Universidad y Salud

ARTÍCULO ORIGINAL

\title{
Ejercicio y educación mejoran equilibrio y la calidad de vida en mujeres con incontinencia urinaria de esfuerzo
}

\author{
Nashari Pérez-Cimma' orcid.org/0000-0001-5785-2894 \\ Igor Cigarroa ${ }^{2}$ orcid.org/0000-0003-0418-8787 \\ Rafael Zapata-Lamana ${ }^{3}$ orcid.org/0000-0002-4729-1680 \\ Sonia Sepúlveda-Martin4 orcid.org/0000-0002-4991-8416 \\ Pamela Espinoza-Pulgar ${ }^{2}$ orcid.org/0000-0002-8713-5885 \\ Carla Sarqui2* orcid.org/0000-0001-7113-5795
}

Exercise and education improve balance and quality of life in women with stress urinary incontinence

1. Centro de Salud y Estética Lipoderma LTDA. Los Ángeles, región del Biobío, Chile.

2. Escuela de kinesiología, Facultad de Salud, Universidad Santo Tomás. Los Ángeles, región del Biobío, Chile.

3. Escuela de Educación, Universidad de Concepción. Los Ángeles, región del Biobío, Chile.

4. Departamento de Ciencias Clínicas y Preclínica, Facultad de Medicina, Universidad Católica de la Santísima Concepción, Concepción, región del Biobío, Chile.

Pérez-Cimma N, Cigarroa I, Zapata-Lamana R, Sepúlveda-Martin S, Espinoza-Pulgar P, Sarqui C. Ejercicio y educación mejoran equilibrio y la calidad de vida en mujeres con incontinencia urinaria. Univ. Salud. 2022;24(1):36-44. DOI: https://doi.org/10.22267/rus.222401.255

\section{Resumen}

Introducción: La incontinencia urinaria por esfuerzo (IUE) tiene una alta prevalencia en mujeres adultas, afectando variables psicológicas, sociales y funcionales como la disminución de capacidad de equilibrio, debido a una escasa contribución en los movimientos del tronco hacia una corrección postural. Objetivo: Determinar los efectos de un programa basado en ejercicio muscular de piso pélvico y educación sobre el equilibrio estático y la calidad de vida en mujeres con IUE. Materiales y métodos: Participaron 18 mujeres con IUE durante 12 semanas en 10 sesiones de ejercicio muscular de piso pélvico y educación (hábitos de higiene, micción, ingesta de líquidos). Pre y post-intervención se evaluó equilibrio estático mediante oscilografía postural y calidad de vida mediante el International Consultation on Incontinence Questionnaire Short-Form (ICQ-SF). Los datos fueron analizados con la prueba no paramétrica de Wilcoxon. Resultados: Se encontró una disminución significativa en el área de desplazamiento del centro de presión en el subtest ojos abiertos $(p=0,027)$ y en el Subtest ojos cerrados $(p=0,006)$. Disminuyó la sintomatología asociada a IUE $(p=0,0001)$. Conclusiones: Pos-intervención mejora equilibrio estático y calidad de vida, confirmando los efectos positivos de este programa que pueden servir de orientación a profesionales de la salud que trabajan con mujeres con IUE.

Palabras clave: Incontinencia urinaria de esfuerzo; piso pélvico; calidad de vida; balance postural. (Fuente: DeCS, Bireme).

\begin{abstract}
Introduction: Stress urinary incontinence (SUI) has high prevalence in adult women, affecting psychological, social and functional variables, including decreased balance capacity, due to a lack of trunk movements that contribute to postural correction. Objective: To determine how a program based on pelvic floor muscle exercises and education affects static balance and quality of life of women with SUI. Materials and methods: 18 SUI female patients participated in a 12 week/10 sessions program that included pelvic floor muscle exercises and education (hygiene habits, urination, fluid intake). Static balance and quality of life were assessed before and after the intervention using postural oscillography and the International Consultation on Incontinence Questionnaire Short Form(ICQ-SF), respectively. Data were analyzed with the non-parametric Wilcoxon test. Results: The displacement area of the center of pressure in the open $(p=0.027)$ and closed $(p=0.006)$ subtests showed a significant reduction. Likewise, the amount of symptoms associated with SUI decreased $(\mathrm{p}=0.0001)$. Conclusions: Intervention improves static balance and quality of life, confirming the positive effects of this program, which can serve as a guide for health professionals who work with women with SUI.
\end{abstract}

Keywords: Stress urinary incontinence; pelvic floor; quality of life; postural balance. (Source: DeCS, Bireme).

\footnotetext{
*Autor de correspondencia

Carla Sarqui

e-mail: csarqui@santotomas.cl
} 


\section{Introducción}

La Sociedad Internacional de la Continencia (ICS) define a la incontinencia urinaria (IU) como la pérdida involuntaria de orina a través de la uretra, objetivable y en cantidad suficiente como para constituir un problema higiénico y social $(1,2)$. Mundialmente, casi el 50\% de las mujeres adultas podrían experimentar $\mathrm{IU}^{(3)}$, dado que existe un aumento considerable del porcentaje a medida que avanza la edad(4-8). Esta patología tiene consecuencias psico-sociales y funcionales que impone cargas significativas en la vida de quien la padece ${ }^{(2,8)}$.

La incontinencia urinaria de esfuerzo (IUE) es la IU más prevalente en mujeres adultas(2,4,9-12). La cual produce una falla en los mecanismos de resistencia uretral, generando la pérdida involuntaria de orina en actividades cotidianas que implican un aumento de la presión intraabdominal; por ejemplo ejercicios de tronco y extremidades, la tos y los estornudos $(6,12)$. Debido a esto, se generan problemas en la marcha, postura y equilibrio, lo que disminuye la calidad de vida de estas pacientes $(7,13)$.

Dentro de los factores de riesgo se encuentran la edad avanzada, sexo femenino, estilos de vida como el sedentarismo, práctica de deportes de alto impacto y consumo de tabaco, multiparidad, parto vaginal, inicio de la menopausia, cirugías genitourinarias y abdominales previas, y factores específicos que aumentan la presión intraabdominal (obesidad, determinados esfuerzos físicos y el estreñimiento)(10,12,14-16).

La obesidad, la multiparidad y el sobreesfuerzo aumentan la presión intraabdominal y la distensión de los músculos abdominales y diástasis abdominal(2,12,17), produciendo un desplazamiento anterior de la línea umbilico-pubiana e hipertransmisión de vectores resultantes hacia la región del periné anterior, zona que progresivamente perderá la capacidad de sostén de los órganos pélvicos, favoreciendo una hipotonía de la musculatura y la aparición de prolapso pélvico e IU(18,19). Por otro lado, el aumento de la actividad del piso pélvico y de los músculos del tronco en mujeres con IUE puede afectar el equilibrio como resultado de una contribución reducida del movimiento del tronco a la corrección postural o la agudeza propioceptiva comprometida. No obstante, se ha encontrado escasa literatura que haya evaluado el equilibrio en esta población(13).
Se ha comprobado que las fibras horizontales del transverso abdominal y sus inserciones en la fascia toracolumbar permiten un control fino de la región lumbo-pélvica por una acción directa sobre las vértebras lumbares y el aumento de la presión intrabdominal. Además, los músculos abdominales superficiales ayudan a la rigidez y juegan un papel dinámico a la hora de generar movimiento para la columna(18,20,21). Sobre la base de la actividad muscular descrita, y considerando que el tratamiento conservador debiera ser el tratamiento de primera línea en pacientes con IU(7), se han diseñado variados tipos de programas basados en ejercicio terapéutico para fortalecer la musculatura tóraco-abdomino pelviana en esta población, tales como el ejercicio aeróbico o de fuerza-resistencia muscular en piso pélvico, siendo estos últimos creados por Arnold Kegel en 1940(22,23) los más utilizados como método para el control de este tipo de incontinencia(23-26).

A pesar de estar reportada la efectividad de los ejercicios musculares de piso pélvico en parámetros funcionales de sujetos con IU(19,26-28), en Chile existen escasos programas gubernamentales orientados a la rehabilitación de esta población y no existe un consenso sobre programas de intervención. Este estudio sería la primera investigación en Chile, que propone un programa de ejercicios musculares de piso pélvico y educación para pacientes con IUE.

Sobre la base de lo expuesto, el objetivo del estudio fue determinar los efectos de una intervención basada en ejercicios musculares de piso pélvico y terapia conductual sobre el equilibrio estático y la calidad de vida en mujeres con IUE.

\section{Materiales y métodos}

\section{Diseño}

Estudio de diseño preexperimental pre-post prueba sin grupo control.

\section{Población y muestra}

Se invitó a participar a 24 mujeres adultas con diagnóstico de médico ginecólogo de IUE leve y moderada. El diagnóstico se hizo a través de la clínica de las pacientes y el cuestionario de Sandvick para determinar el nivel de incontinencia (1-2=leve; 3$6=$ moderada; $8-9=$ grave y $12=$ muy grave) ${ }^{(29)}$. Se utilizó una muestra por conveniencia y un muestreo de sujetos voluntarios que cumplieran con los siguientes criterios de inclusión y exclusión. 


\section{Criterios de inclusión}

Se incluyó a mujeres entre 40 y 60 años diagnosticadas con IUE entre agosto y noviembre del año 2016 y que fueron derivadas al Servicio de Medicina Física y Rehabilitación de un Complejo hospitalario de una ciudad del sur de Chile.

\section{Criterios de exclusión}

Se excluyeron a mujeres con diagnóstico médico de IUE con presencia de alteraciones vestibulares, auditivas, visuales y propioceptivas que alteran la estabilidad, con presencia de trastornos músculo esqueléticos, dolor lumbar agudo o crónico, cuadros neurológicos que dificultaran la marcha $y$ bipedestación $(n=4)$, presencia de patología psiquiátrica que le impidiera tomar decisiones por sí misma o seguir instrucciones dadas por el examinador $(n=1)$, embarazadas $(n=1)$. Finalmente, se excluyó un total de 6 mujeres, así, la muestra final quedó constituida por 18 mujeres. Se generó un único grupo experimental que realizó el programa de ejercicios musculares de piso pélvico y educación conductual.

\section{Procedimientos}

La intervención se realizó en diez sesiones durante 12 semanas utilizando los siguientes procedimientos y distribución:

\section{Educación}

Durante todas las sesiones se realizó educación en relación al cambio en los estilos de vida, tanto en alimentación para evitar el aumento de peso, cese del hábito tabáquico. Adicionalmente se educó sobre actividades de la vida diaria y hábitos saludables tales como, realizar micciones programadas y disminuir la ingesta de líquidos, higiene adecuada en la zona vaginal y perianal $(30,31)$.

\section{Programa de ejercicio}

Semanas 1 y 2 (dos sesiones a la semana): En la primera sesión se trabajó el reconocimiento de estructuras y activación muscular del suelo pélvico. Desde la segunda a la cuarta sesión se realizó una valoración manométrica de la capacidad contráctil de la musculatura utilizando la escala de $\operatorname{Oxford}^{(29,32)}$ y Biofeedback (Enraf Nonius- Myomed 632X) para objetivar y/o modificar la actividad muscular en los casos en que la contracción no era efectiva(26).

Además, se realizó entrenamiento muscular y reeducación funcional del piso pélvico (trabajo de localización y disociación) y se enseñó ejercicios de activación de la musculatura con contracción consciente antes y durante el esfuerzo para evitar la pérdida de orina(26). Con el objetivo de fortalecer la musculatura se realizaron 1 a 2 series de 10 repeticiones de contracciones voluntarias máximas, manteniéndolas durante aproximadamente 6 segundos y haciendo una pausa de 6 segundos entre cada una de ellas. La recuperación entre series fue de 1 a 3 minutos. Las contracciones se llevaban a cabo

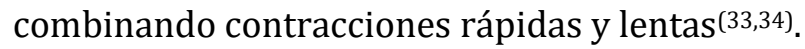

Semanas 3 y 4 (una sesión a la semana): Desde la quinta sesión se inició la ejercitación de la musculatura de piso pélvico ante la tos y esfuerzos mediante la técnica de bloqueo perineal y ejercicios de activación de los músculos abdominales, especialmente el transverso abdominal. Se trabajó coordinación, fuerza, resistencia y velocidad de contracción muscular asociado al entrenamiento funcional y propioceptivo.

Semanas 5 a 8 (dos sesiones cada mes) y Semanas 9 a 12 (una sesión cada mes): A partir de la sexta hasta la décima sesión, se reforzó la función de sostén activando los distintos tipos de fibras musculares que conforman el piso pélvico(26) para conseguir un adecuado tono perineal y asegurar la continencia urinaria durante los esfuerzos. En ambos casos se realizaron ejercicios sobre balón terapéutico y uso de bandas elásticas con resistencia gradual, asociadas a la respiración y contracción de la musculatura abdomino pélvica. Se mantuvo la serie de contracciones voluntarias máximas mantenidas (Figura 1). 


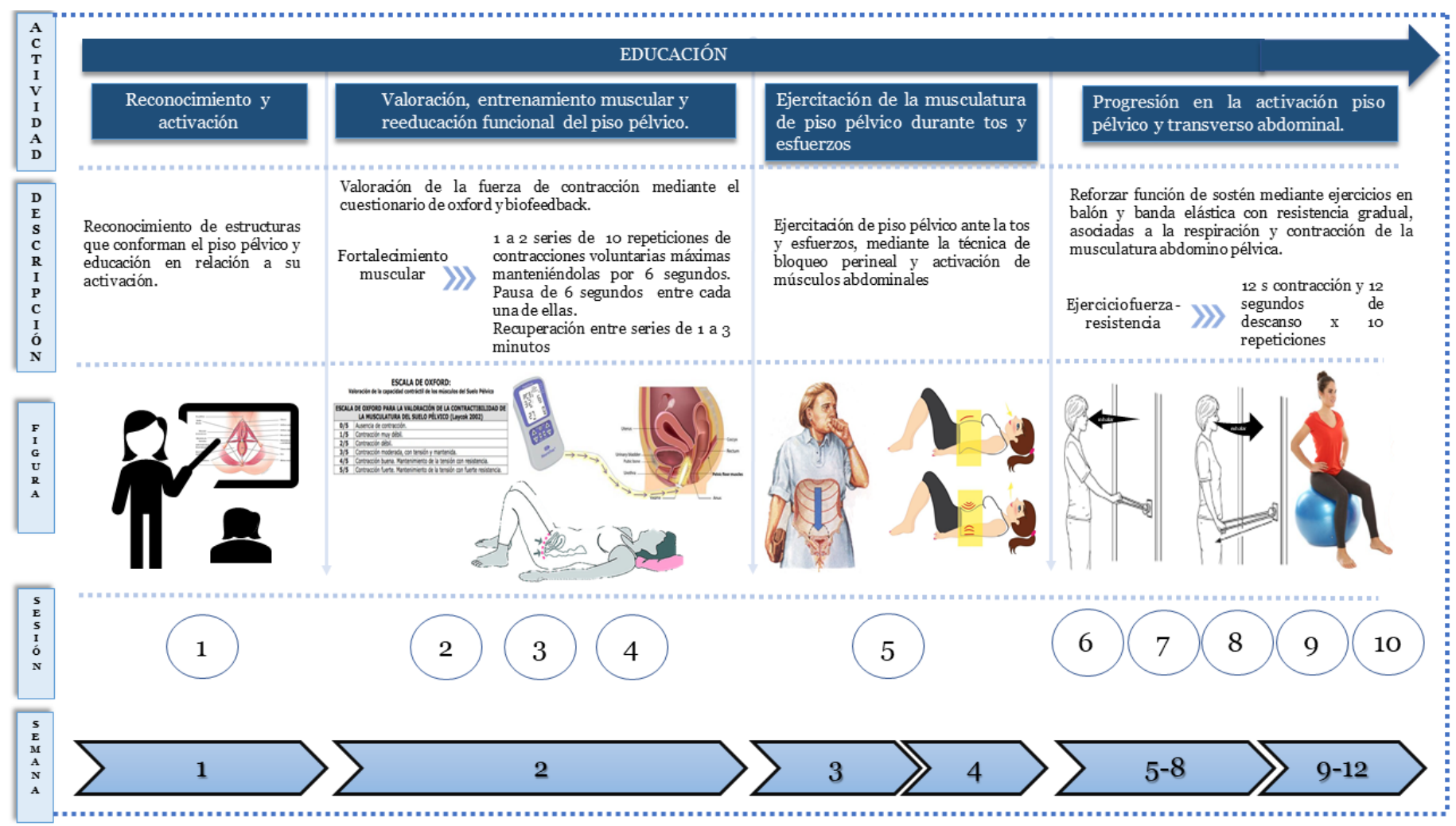

Figura 1. Programa de ejercicio y educación durante las 12 semanas de entrenamiento.

Semanas 13 a 20 (una sesión cada dos meses): Adicionalmente, se realizó seguimiento y control con el objetivo de supervisar la correcta ejecución de los ejercicios y mantener el trabajo individual en el hogar. La indicación fue realizar los ejercicios tres veces al día al menos tres días a la semana(33,34).

\section{Variables de análisis Equilibrio estático}

Se realizó la evaluación utilizando un oscilógrafo postural marca artOficio con sensibilidad de 0,1 Newton, utilizado comúnmente en el campo del control motor y balance postural(35,36). Cada sujeto debía mantenerse lo más quieto posible en posición bípeda erguida y descalzo sobre la plataforma completando dos subtest de 30 segundos (vista al frente y ojos cerrados) ${ }^{(35,36)}$. En el subtest vista al frente se buscó la situación más natural de la postura basada en los estímulos propioceptivos, visual y vestibular. Se pidió al sujeto que fijara su mirada en un blanco ubicado en una pared a nivel de los ojos y a $3 \mathrm{~m}$ de distancia.

En el subtest ojos cerrados se buscó la respuesta de los sistemas propioceptivo y vestibular y la condición de mantenerse erguido sin abrir los ojos debía ser observada por el examinador. El lugar se encontraba libre de distractores visuales y auditivos y se resguardó la seguridad del sujeto durante todo el tiempo que duró la prueba. Las variables posturográficas analizadas fueron velocidad media de desplazamiento del centro de presiones (VmCOP) y el área de desplazamiento del centro de presiones (AdCOP)(37).

\section{Calidad de vida}

Se aplicó el International Consultation on Incontinence Questionnaire Short-Form (ICQ-SF) ampliamente usado para la evaluación los síntomas y el impacto de la IU en la calidad de vida de las participantes ${ }^{(38)}$. El ICIQ-SF es un cuestionario breve y simple validado en Chile( ${ }^{(39)}$. Su utilización puede hacerse extensiva a toda la población y es de gran utilidad a las diversas unidades de uroginecología existentes en el país. La valoración de la calidad de vida se midió por la puntuación total obtenida, donde 0 puntos es buena calidad de vida y 20 puntos mala calidad de vida.

Ambas evaluaciones se desarrollaron en el laboratorio de análisis del movimiento humano de una universidad, el cual cumple con condiciones estándar de luminosidad, temperatura, humedad y 
aislamiento acústico y fueron realizadas por un profesional de la salud con experiencia en la toma de datos de estas variables. El programa de ejercicio y la educación fueron realizados en un complejo hospitalario por otro profesional de la salud con experiencia en prescripción de ejercicio físico en mujeres con IUE; este profesional fue diferente al que hizo las evaluaciones y así se evitó el sesgo de detección.

\section{Consideraciones éticas}

Las evaluaciones, programa de ejercicio y educación fueron aprobados por el Comité Ético Científico de un Servicio de Salud de la zona sur de Chile y se realizaron previa firma de consentimiento informado. Todos los procedimientos siguieron los principios éticos para investigaciones médicas en seres humanos de acuerdo con la Declaración de Helsinki actualizada en Fortaleza, Brasil (2013).

\section{Análisis estadístico}

El análisis descriptivo se expresó en mediana e IC 95\%. Para determinar la normalidad de las variables dependientes se utilizó prueba de Shapiro-Wilk. Posteriormente, para establecer diferencias pre-post tratamiento se utilizó la prueba no paramétrica de Wilcoxon. El nivel de significancia utilizado en el pretest y postest respectivamente fue ${ }^{*} p<0,05 \mathrm{y}$ ${ }^{* *} p<0,01$. El análisis estadístico se hizo con el programa SPSS v.19.0.

\section{Resultados}

Las mujeres con IUE tuvieron una mediana de 50,5 años, un peso de $68 \mathrm{~kg}$ y un Índice de Masa Corporal (IMC) de 29,7, lo que indica sobrepeso. La intervención propuesta basada en ejercicios musculares de piso pélvico y terapia conductual tuvo una duración de 12 semanas, de 10 sesiones. En cada sesión se realizó 45 minutos de ejercicios y 5 minutos de educación. Esta intervención tuvo un 100\% de adherencia y $0 \%$ de retiros o lesionados (Tabla 1 ).
Asimismo, se comparó las variables de equilibrio estático en condiciones de ojos abiertos y cerrados pre y post ejercicio. Se evidenció que las mujeres con IUE disminuyeron significativamente el área de desplazamiento del centro de presiones (COP) tanto en condiciones de ojos abiertos como cerrados, por lo tanto, se observó un mejor equilibrio estático post intervención (Tabla 2).

Para evaluar los efectos producidos por la intervención en la calidad de vida se aplicó el cuestionario de calidad de vida ICQ-SF. La Tabla 2 compara el puntaje obtenido en el cuestionario de calidad de vida pre y post ejercicio. La prueba $\mathrm{T}$ evidenció que el ejercicio disminuyó la cantidad de síntomas asociados a la IUE, lo que indicó una mejora en la calidad de vida de estos sujetos. En la evaluación previa al inicio de los ejercicios y al finalizar las 10 sesiones no se observaron diferencias significativas en la frecuencia cardiaca, saturación de oxígeno e índice de esfuerzo de Borg (Tabla 3).

Tabla 1. Características de la muestra y la intervención

\begin{tabular}{lrr}
\hline \multicolumn{1}{c}{ Variables clínicas } & Mediana & \multicolumn{1}{c}{ IC 95\% } \\
\hline Edad & 50,5 & {$[48,69-54,75]$} \\
Peso (kg) & 68,0 & {$[66,74-78,15]$} \\
Estatura (cm) & 155,3 & {$[152,21-$} \\
& & $157,79]$ \\
IMC (talla/kg2) & 29,7 & {$[28,12-32,52]$} \\
№ de hijos & 2,5 & {$[2,31-3,13]$} \\
Nivel de incontinencia & 3,2 & {$[3,01-3,43]$} \\
(cuestionario Sandvick) & & \\
Características de la & & \\
intervención & & \\
Tiempo de intervención & 20 & \\
(semanas) & & \\
Cantidad de sesiones (número) & 10 & \\
$\begin{array}{l}\text { Duración de sesión de ejercicio } \\
\text { (minutos x sesión) }\end{array}$ & 45 & \\
$\begin{array}{l}\text { Duración de educación } \\
\text { (minutos x sesión) }\end{array}$ & & \\
\hline
\end{tabular}

Tabla 2. Efectos del programa de ejercicio y terapia conductual en el equilibrio estático y calidad de vida

\begin{tabular}{|c|c|c|c|c|}
\hline \multicolumn{2}{|r|}{ Equilibrio estático } & \multirow{2}{*}{ Pre-programa } & \multirow{2}{*}{ Post-programa } & \multirow[b]{2}{*}{$p$-valor } \\
\hline Condición & Variable & & & \\
\hline \multirow[t]{2}{*}{$\mathrm{OA}$} & VmCOP (mm/s) & $228,5[226,08-233,70]$ & $231,0[228,83-236,39]$ & 0,297 \\
\hline & $\operatorname{AdCOP}\left(\mathrm{mm}^{2}\right)$ & $10,5[8,21-13,79]$ & $6,0[3,20-14,91]$ & $0,027^{* *}$ \\
\hline \multirow[t]{2}{*}{$\mathrm{OC}$} & VmCOP (mm/s) & $234,0[233,25-242,30]$ & $238,5[233,04-248,18]$ & 0,430 \\
\hline & $\operatorname{AdCOP}\left(\mathrm{mm}^{2}\right)$ & $12,0[11,17-16,94]$ & $6,0[45,00-13,49]$ & $0,006^{*}$ \\
\hline \multicolumn{5}{|c|}{ Calidad de vida } \\
\hline Puntaje total & cuestionario de calidad de vida ICQ-SF & $13,0[11,25-14,97]$ & $7,0[5,65-9,24]$ & $0,000^{*}$ \\
\hline
\end{tabular}

Los valores son presentados en media e IC 95\%. Donde Vm: Velocidad media de desplazamiento, Ad: Área de desplazamiento, COP: Centro de presión, OA: Ojos abiertos, OC: Ojos cerrados. ${ }^{*} p<0.05 ;{ }^{* *} p<0,01 . \mathrm{n}=18$. 
Tabla 3. Medición de variables de control pre y post intervención

\begin{tabular}{lrr}
\hline \multicolumn{1}{c}{ Variables } & Pre-sesión & \multicolumn{1}{c}{ Post-sesión } \\
\hline Frecuencia cardiaca (latidos x minuto) & $82,0[81,17-82,72]$ & $82,0[81,80-83,09]$ \\
Saturación de oxígeno (\%) & $98,0[97,13-97,98]$ & $9,0,326$ \\
Índice de esfuerzo de Borg & $0,0[-0,06-0,17]$ & $0,07,00-97,78]$ \\
\hline
\end{tabular}

Los valores son presentados en media e IC 95\%. $n=18$.

\section{Discusión}

Los principales resultados del estudio sugieren que las mujeres con IUE mejoraron su calidad de vida y el equilibrio estático medido a través del AdCOP después de haber realizado un programa de ejercicios musculares de piso pélvico y terapia conductual. Se observó que la variable AdCOP, tanto en condición ojos abiertos como cerrados disminuye post ejercicio muscular de piso pélvico, lo que sugiere un mejor control postural estático de estas mujeres. Resultados similares ya habían sido encontrados en población de mujeres adultas con obesidad en donde se evidenció que las mujeres con IU tienen una capacidad de equilibrio disminuida en comparación a mujeres sin IU, la diferencia en relación al presente estudio es que se evaluó con la vejiga llena y vacía, y, además, en mujeres con y sin IU(40). Se sugiere que un programa de ejercicio que estimula la estabilización de la musculatura del $\operatorname{CORE}^{(20)}$ generaría un menor tiempo de respuesta en el reclutamiento del transverso del abdomen y, por lo tanto, una mejor estabilidad postural $(20,40)$. Por otro lado, en el comportamiento de la variable VmCOP no se evidencian cambios significativos post ejercicio tanto para la fase de ojos abiertos como ojos cerrados.

Se observa que las mujeres con IUE disminuyen sus puntajes finales en el cuestionario ICIQ-SF su calidad de vida posterior a la intervención, lo que sugiere un impacto positivo en la calidad de vida de estos pacientes. Resultados similares fueron encontrados por Carneiro et al.(27), quienes evaluaron mujeres con IUE ( $n=50 ; 40-50$ años) el efecto de un programa de ejercicio en la movilidad del cuello vesical, espesor de los músculos del suelo pélvico, fuerza muscular y la calidad de vida con escala ICIQ-SF(28). Hubo mejoras en las características anatomo-funcionales del suelo pélvico y una reducción de 6 puntos promedio en la escala ICIQ-SF en quienes realizaron ejercicios perineales, lo que repercute positivamente en la calidad de vida.

Las principales fortalezas del estudio radican en haber utilizado un oscilógrafo postural para medir objetivamente el equilibrio estático. A diferencia de estudios previos ${ }^{(41,42)}$ que evaluaron el equilibrio a través de pruebas clínicas como el timed up and go o la estación unipodal que son escalas semicuantitativas, subjetivas y menos sensibles a los cambios clínicos en comparación con el análisis instrumentado(43).

¿Qué aporta este estudio a la ciencia y disciplina? Es la primera investigación en Chile que estudia el equilibrio estático en pacientes con IUE, problemática de salud pública cuya prevalencia ha aumentado en este país(44). Sobre la base de los hallazgos se hace relevante la inclusión de intervenciones y evaluaciones que consideren el control postural en estos pacientes, ya que los músculos del suelo pélvico y transverso abdominal se activan en forma primaria para mantener una postura adecuada y continencia urinaria ante los cambios de presiones que se producen en el compartimento abdomino-pelviano. Un programa de ejercicios que considere un buen control del equilibrio no solo podría evitar incontinencias urinarias, sino también la aparición de eventuales prolapsos en esta población.

\section{Limitaciones}

Entre las limitaciones, se mencionan su pequeña muestra $(n=18)$ y la ausencia de grupo control, como era un estudio piloto, el objetivo era evaluar beneficios pre-post intervención antes de realizar un estudio controlado y aleatorizado, por lo que no se puede asegurar que los cambios observados sean debidos al programa de ejercicios musculares de piso pélvico y terapia conductual, o a otras intervenciones o factores no controlados.

Otra debilidad fue no controlar parámetros de estilos de vida relacionados con la salud como sedentarismo o práctica de deporte. Así como datos sobre el tipo de parto, estreñimiento, fuerza muscular, capacidad funcional, riesgo de caídas o presencia de molestias musculoesqueléticas como los han reportado estudios previos ${ }^{(9,12)}$. Por esto no fue posible concluir si este programa, además de generar efectos positivos en los parámetros estudiados, pudo generar beneficios a nivel muscular, funcional, clínico(13) y 
psicosocial o, por el contrario, generar efectos negativos.

En este sentido, se recomiendan estudios que consideren mayor número de variables y con un diseño experimental que permitan determinar el impacto de este tipo de intervenciones en la salud integral de pacientes con IUE. Adicionalmente, en la evaluación del equilibrio estático se debería haber considerado el estado de la vejiga. Los músculos del piso pélvico además de mantener la continencia participan en el control postural de la columna, por lo tanto, a medida que aumenta el llenado de la vejiga, estos músculos se ven sometidos a un desafío mayor, lo que puede producir una pérdida de continencia o del control postural.

\section{Conclusiones}

Un programa de 12 semanas de fortalecimiento de la musculatura de piso pélvico y de educación, disminuyó el AdCOP, lo que sugiere una mejora en el equilibrio estático. Además, se disminuyó la sintomatología asociada a la IUE, lo que indicaría una mejora en la calidad de vida de estas mujeres.

Se ha creado una intervención segura, adaptada a las condiciones clínicas de los pacientes, con excelente adherencia, sin lesionados ni efectos desfavorables posteriores a su ejecución que podría servir de punto de partida para abordar la rehabilitación de esta problemática de salud pública cada vez más prevalente en la población de mujeres. Estos hallazgos confirman los efectos positivos de este programa de ejercicios y educación y puede servir de orientación a profesionales de la salud que trabajan con mujeres con IUE.

Financiación: La presente investigación no ha recibido ayudas específicas provenientes de agencias del sector público, sector comercial o entidades sin ánimo de lucro.

Conflicto de intereses: Ninguno declarado por los autores.

\section{Referencias}

1. Rogers RG, Pauls RN, Thakar R, Morin M, Kuhn A, Petri E, et al. An International Urogynecological Association (IUGA)/International Continence Society (ICS) joint report on the terminology for the assessment of sexual health of women with pelvic floor dysfunction. Neurourol Urodyn [Internet]. 2018 Apr 14;37(4):1220-40. Disponible en: https://onlinelibrary.wiley.com/doi/abs/10.1002/nau.235 08

2. Castañeda BI, Martínez TJC, García DJÁ, Rodríguez AEM, Pérez RNM. Aspectos epidemiológicos de la incontinencia urinaria. Revisión Bibliográfica. Rev Cuba Med Física y Rehabil. 2016;8(Suppl 1):88-98. Disponible en: https://www.medigraphic.com/cgi-

bin/new/resumen.cgi?IDARTICULO=72108

3. González-Ruíz C, Pérez-Hero ML, Jalón-Monzón A, GarcíaRodríguez J. Actualización en incontinencia urinaria femenina. Semergen. 2017;43(8):578-84. DOI: 10.1016/j.semerg.2017.01.003.

4. González-Maldonado LA, Erosa-Villarreal RA, JanssenAguilar R, Laviada-Molina HA, Méndez-Domínguez NI. Urinary incontinence: Risk factors and frequency in women above 60 years of age in the Southeast of Mexico. Rev Mex Urol. 2019;79(3):e03. Disponible en: http://www.scielo.org.mx/scielo.php?script=sci_arttext\&pi $\mathrm{d}=\mathrm{S} 2007-40852019000300003$

5. Tuda C, Carnero MP. Prevalencia y factores asociados a incontinencia urinaria en el área de salud este de Valladolid. Enferm glob. 2020;19(1):390-412. Disponible en: https://scielo.isciii.es/scielo.php?script=sci_arttext\&pid=S1 695-61412020000100012\&lng=es

6. Lukacz ES, Santiago-Lastra Y, Albo M, Brubaker L. Incontinencia urinaria en mujeres Una revisión. JamaJournal Am Med Assoc. 2017;318(16):1592-16054. DOI: 10.1001/jama.2017.12137.

7. Da Costa A, Vasconcellos I, Pacheco R, Di Bella Z, Riera R. What do Cochrane systematic reviews say about nonsurgical interventions for urinary in-continence in women? Sao Paulo Med J. 2018;136(1):73-83. DOI: 10.1590/15163180.2017.039420122017.

8. Gretchen I. Urinary Incontinence. Prim Care. 2019;46(2):233-42. DOI: 10.1016/j.pop.2019.02.004.

9. Oliveira Alves J, Tonon Da Luz S, Brandão S, Madeiros Da Luz C, Natal Jorge R, Da Rosa T. Urinary Incontinence in Physically Active Young Women: Prevalence and Related Factors. Int J Sports Med. 2017;38(12):937-41. DOI: 10.1055/s-0043-115736.

10. Capobianco G, Madonia M, Morelli S, Dessole F, De Vita D, Cherchi $\mathrm{P}$, et al. Management of female stress urinary incontinence: A care pathway and update. Maturitas. 2018;109:32-8. DOI: 10.1016/j.maturitas.2017.12.008.

11. Pazmiño LM, Esparza D, Ayala L, Quinteros MJ. Prevalencia de la incontinencia urinaria en mujeres de 45-65 años del Hospital Padre Carollo. Mediciencias UTA. 2019;3(2):69-75. DOI: 10.31243/mdc.uta.v3i2.168.2019.

12. Da Roza T, Brandao S, Mascarenhas T, Jorge R, Duarte J. Urinary Incontinence and Levels of Regular Physical Exercise in Young Women. Int J Sports Med. 2015;36(9):776-80. DOI: 10.1055/s-0034-1398625.

13. Chmielewska D, Stania M, Słomka K, Błaszczak E, Taradaj J, Dolibog P, et al. Static postural stability in women with stress urinary incontinence: Effects of vision and bladder filling. Neurourology and Urodynamics. Neurourol Urodyn. 2017;36(8):2019-27. DOI: 10.1002/nau.23222.

14. Campani Nygaard C, Schereiner L, Picolli Morsch T, Petersen Saadi R, Faria Figueiredo M, Vontobel Padoin A. Urinary Incontinence and Quality of Life in Female Patients with Obesity. Rev Bras Ginecol Obstet. 2018;40(9):534-9. DOI: 10.1055/s-0038-1670626. 
15. Nygaard I, Shaw J. Physical activity and the pelvic floor. Am J Obstet Gynecol. 2016;214(2):164-71. DOI: 10.1016/j.ajog.2015.08.067.

16. Téllez-Díaz JA, Aragón-Castro MA, Vázquez-Niño LC, Gutiérrez-Rosales R, Ruvalcaba-Oceguera GE, GuerreroReyes G, et al. Aspectos actuales en el tratamiento de la incontinencia urinaria de esfuerzo en mujeres. Rev Mex Urol. 2017;77(5):411-8. Disponible en: https://www.medigraphic.com/pdfs/uro/ur2017/ur175k.pdf

17. Cañamero de León S, Da Cuña Carrera I, De la Hoz González C, Soto Gonzáles M. Comparación De Los Efectos De Un Programa De Ejercicio Sobre Diastasis De Rectos Según El PeRiodo Postparto. Med Natur [Internet]. 2020;14(1):53-8. Disponible en: https://dialnet.unirioja.es/servlet/articulo? codigo=724897 6

18. Madokoro S, Miaki H. Relationship between transversus abdominis muscle thickness and urinary incontinence in females at 2 months postpartum. J Phys Ther Sci. 2019;31(1):108-11. DOI: 10.1589/jpts.31.108.

19. Alewijnse D, Metsemakers J, Mesters I, Van den Borne B. Effectiveness of pelvic floor muscle exercise therapy supplemented with a health education program to promote long-term adherence among women with urinary incontinence. Neurourol Urodyn. 2003;22(4):284-95. DOI: 10.1002/nau.10122.

20. Segarra V, Heredia JR, Peña G, Sampietro M, Moyano M, Mata $F$, et al. Core y sistema de control neuro-motor: mecanismos básicos para la estabilidad del raquis lumbar. Rev Bras Educ Fís Esporte. 2014;28(3):521-9. DOI: 10.1590/S180755092014005000005.

21. Sapsford R. Rehabilitation of pelvic floor muscles utilizing trunk stabilization. Man Ther. 2004;9(1):3-12. DOI: 10.1016/s1356-689x(03)00131-0.

22. Kegel A. Progressive resistance exercise in the functional restoration of the perineal muscles. Obs Gynecol. 1948;56(2):238-48. DOI: 10.1016/0002-9378(48)90266-x.

23. Cavkaytar S, Kokanali MK, Topcu HO, Aksakal OS, Doğanay M. Effect of home-based Kegel exercises on quality of life in women with stress and mixed urinary incontinence. J Obs Gynaecol. 2015;35(4):407-10. DOI: 10.3109/01443615.2014.960831.

24. Chang Calderin O, Pérez Rodríguez M, Figueredo Villa K, Torres Pérez M, Torres Pérez M, Llori Otero K. Efectividad de la reeducación del suelo pélvico en adultas mayores con incontinencia urinaria de esfuerzo. Rev Cuba Med Gen Integr. 2019;35(1):e785. Disponible en: https://www.medigraphic.com/cgi-

bin/new/resumen.cgi?IDARTICULO=91084

25. Aranda Lozano J, Sierra Labarta R. Incontinencia urinaria de esfuerzo en mujeres: impacto en la calidad de vida tras rehabilitación del suelo pélvico. Rev Arg de Urol. 2018;83(2):60-7. Disponible en: https://www.revistasau.org/index.php/revista/article/vie wFile/4147/3545

26. Fuentes B, Venegas $M$. The Role of a physiotherapist in the pelvic floor unit. Rev Med Clin Condes. 2013;24(2):305-12. Disponible en: https://www.clinicalascondes.cl/Dev_CLC/media/Imagene s/PDF\%20revista\%20m\%C3\%A9dica/2013/2\%20marzo/ 14-Kine.Fuentes.pdf

27. Carneiro E, Araujo S, Beuttenmüll L, Vieira P, Cader S, Rett M, et al. The anatomical-functional characteristics of the pelvic floor and quality of life of women with stress urinary incontinence subjected to perineal exercises. Actas Urol Esp. 2010;34(9):788-93. Disponible en: https://pubmed.ncbi.nlm.nih.gov/20843456/

28. Castro Adrada LM. Entrenamiento del suelo pélvico en incontinencia urinaria durante el embarazo y postparto vaginal. FisioGlia [Internet]. 2017;4(3):53-6. Disponible en: https://dialnet.unirioja.es/servlet/articulo?codigo $=608496$ 2

29. Laycock J. Patient Assessment. In: Laycock J, Haslam J (eds). Therapeutic Management of Incontinence and Pelvic Pain. London: Springer; 2002. DOI: 10.1007/978-1-4471-37153_6.

30. Oteo Manjavacas P, Donis Canet F, Estigaribia Benítez CA, Costell J. Incontinencia urinaria en la mujer: evaluación y manejo práctico en atención primaria. Form medica Contin en atención primaria. 2020;27(9):480-7. DOI: 10.1016/j.fmc.2020.05.002.

31. Manual de protocolos y procedimientos generales de enfermería. Higiene perianal y cuidados en la incontinencia [Internet]. Hospital universitario Reina Sofía; 2010. 8 p. Disponible en: http://www.sspa.juntadeandalucia.es/servicioandaluzdesal ud/hrs3/fileadmin/user_upload/area_enfermeria/enferme ria/procedimientos/procedimientos_2012/b5_higienes_per ianal.pdf

32. Gonazález Sánchez B, Rodríguez-Mansilla J, de Toro García A, González López-Arza MV. Eficacia del entrenamiento de la musculatura del suelo pélvico en incontinencia urinaria femenina. Anales Sis San Navarra [Internet]. 2014;37(3):381-400. DOI: 10.4321/S113766272014000300008.

33. Marques A, Stothers L, Macnab A, Frcpch F. The status of pelvic floor muscle training for women. Can Urol Assoc J. 2010;4(6):419-24. DOI: 10.5489/cuaj.963.

34. García-Sánchez E, Ávila-Gandía V, López-Román J, MartínezRodríguez A, Rubio-Arias JÁ. What Pelvic Floor Muscle Training Load is Optimal in Minimizing Urine Loss in Women with Stress Urinary Incontinence? A Systematic Review and Meta-Analysis. Int $J$ Environ Res Public Health. 2019;16(22):4358. DOI: 10.3390/ijerph16224358.

35. Caniuqueo A, Fernandes J, Quiroz G, Rivas R. Cinética de marcha, balance postural e índice masa corporal durante el primer, segundo y tecer trimestre de embarazo. Rev Peru Ginecol y Obstet. 2014;60(2):109-16. Disponible en: http://www.scielo.org.pe/pdf/rgo/v60n2/a03v60n2.pdf

36. Gatica VF, Velásquez SI, Méndez GA, Guzmán EE, Manterola CG. Differences in standing balance in patients with cerebral palsy and typically developing children. Diferencias en el balance de pie en pacientes con parálisis cerebral y niños con desarrollo típico. Biomédica. 2014;34(1):102-9. DOI: 10.7705/biomedica.v34i1.1535.

37. Duarte M, Freitas S. Revision of posturography based on force plate for balance evaluation. Rev Bras Fisioter. 2010;14(3):183-92. Disponible en: https://www.scielo.br/j/rbfis/a/hFQTppgw4q3jGBCDKV9f $\mathrm{dCH} /$ ?format=pdf\&lang=en

38. Klovning A, Avery K, Sandvik H, Hunskaar S. Comparison of Two Questionnaires for Assessing the Severity of Urinary Incontinence: The ICIQ-UI SF Versus the Incontinence Severity Index. Neurourol Urodyn. 2009;28(5):411-5. DOI: 10.1002/nau.20674.

39. Busquets M, Serra R. Validation of a Spanish version of the International Consultation on Incontinence Ques-tionnaire 
Short-Form. Rev Med Chile. 2012;140(3):340-6. DOI: 10.4067/S0034-98872012000300009.

40. Smith M, Coppieters M, Hodges P. Is balance different in women with and without stress urinary incontinence? Neurourol Urodyn. 2008;27(1):71-8. DOI: 10.1002/nau.20476.

41. Le Berre M, Morin M, Corriveau H, Hamel M, Nadeau S, Filiatrault J, et al. Characteristics of Lower Limb Muscle Strength, Balance, Mobility, and Function in Older Women with Urge and Mixed Urinary Incontinence: An Observational Pilot Study. Physiother Can. 2019;71(3):250-60. DOI: 10.3138/ptc.2018-30.

42. Le Berre M, Dumoulin C. Force, équilibre, mobilité et fonction des femmes âgées avec incontinence urinaire: une revue de la littérature. Kinésithérapie, la Revue. 2020;20(226):3-20. DOI: $10.1016 /$ j.kine.2020.05.020.

43. Ortuño-Cortés MA, Martín-Sanz E, Barona-de Guzmán R. Posturografía estática frente a pruebas clínicas en ancianos con vestibulopatía. Acta Otorrinolaringol Esp. 2008;59(7):334-40. DOI: 10.1016/S0001-6519(08)755523.

44. Rincón Ardila 0. Prevalence and risk factors for urinary incontinence among women consulting in primary care. Rev Med Chil [Internet]. 2015;143(2):203-12. DOI: 10.4067/S0034-98872015000200008 